\title{
Experimental Evaluation of Machine learning based MIMO-OFDM System for Optimal PAPR and BER
}

\author{
SHOBHA Y. K ${ }^{1}$, RANGARAJU H. G ${ }^{2}$ \\ ${ }^{l}$ Department of Electronics \& Communication Engineering, Bahubali College of Engineering, \\ Shravanabelagola, Karnataka, INDIA \\ ${ }^{2}$ Department of Electronics \& Communication Engineering, \\ Govt. SKSJ Technological Institute, Bengaluru, INDIA
}

\begin{abstract}
The hypothetically convenient structure is the Multiple-Input Multiple-Output Orthogonal Frequency Division Multiplexing (MIMO-OFDM) technique that is employed for upcoming generations in wireless communication systems. Some of the benefits offered by MIMO-OFDM are enhanced spatial multiplexing, reliability and network throughput, and so on. Due to the integration of spatial antenna that is based on multistream, the problems which are related to significantly high power takes place in the system of OFDM and provides complex processing strategies. Some of the popularly known systems that are used for standardizing the Peak to average power ratio (PAPR) are partial transmit sequences (PTS), adoptive tone reservation (ATR), probabilistic mapping, and clipping which are required to be truncated and aims for minimizing the operational cost. The framework of hybrid Selective Mapping (SLM)-PTS proposed in this paper minimizes the operational cost by integrating strategies of PTS and SLM. A reduction approach that is suitable for PAPR and BER are chosen for optimization purposes depending on the statistical threshold constraint of PAPR and Bit Error Rate (BER). Thus, the system preferred with the help of the machine learning technique demonstrates the efficiency in implementing a generalized strategy to evaluate a low complexity MIMO-OFDM model. Ultimately, with the help of the PAPR and BER techniques-driven from value bound the performance of the error rate is evaluated in this framework that interactively changes from one technique.
\end{abstract}

Key-Words: - ATR, MIMO, OFDM, PTS, SLM, SVM, 5G and Machine Learning

Received: November 5, 2020. Revised: April 28, 2021. Accepted: May 14, 2021. Published: May 26, 2021.

\section{Introduction}

Orthogonal frequency division multiplexing (OFDM) is regarded as a frequently chosen technique in the system of wireless communication. Since OFDM possess few advantages such as efficiency and reactivity to the frequency of the specific channel, this technique is chosen. Some of the disadvantages of OFDM are out-of-band radiation and the requirement of intricate non-linear power amplifiers due to the large peak-to-average power ratio (PAPR) in the time domain because of the accumulation of phase among various carriers during the mapping process of subcarrier. To obtain time selective fading channels and for obtaining the capacity of the channel, the space-frequency block coding (SFBC) [1] that is included in multiple-input, multiple-output (MIMO) is combined with the system of OFDM. The linear power amplifier is constantly operated by PAPR that significantly high and sometimes generates the features of nonlinearity. Due to non-linear features, few external noises like out-of-band radiation takes place and the specified state of operation is not well suited for optimization thereby computational cost is also significantly more for the functioning of PA in the linear region. Several approaches that are meant for the reduction of PAPR 
are designed to maximize the productivity of PA without inducing a high error rate for the OFDM model. But the techniques that are designed to maximize the efficiency possess few drawbacks at the cost of BER system efficiency [2]. While evaluating the methods which are used for reducing PAPR, the computation cost and BER must be minimum. Depending on the least probable value obtained for the PAPR and also on the mean to determine the best of these constraints the entire performance of the PAPR system is evaluated.

\section{5 HDMGT: RUNV}

Some of the approaches that are used for reducing PAPR are selected mapping (SLM) and partial transmitted sequence (PTS) with finite computational overhead because of its iterative process are examined for the OFDM models. However, the techniques employed for reducing PAPR is integrated with the systems of MIMO-OFDM and results in intricacy drawbacks by enhancing the iteration process. The SLM system is considered as a highly regarded approach for obtaining reduced PAPR with minimum data rate because few bits are reserved and are transmitted as side information (SI) for analyzing the patterns of phase alignment. The side information transmitted results in a lack of QoS and intrusions for a chosen fading channel. The entire fast Fourier transforms (IFFTs) modules are employed for SLM by making use of time-domain signal relations between antenna's that are spatial [5] and are also employed to reduce the complexity in the SFBC MIMO-OFDM model. A predominantly high data rate plays a vital part in multi-carrier systems and must effectively operate in case of significantly high data transmission and mobility including high carrier frequency. Thus, from the research work, it is demonstrated that the OFDM fulfills the criteria of multi-carrier systems. One of the techniques of multicarrier modulation (MCM) is OFDM. The representation of information in this approach is difficult and are namely QAM, QPSK, BPSK, MPSK, and so on. The data symbols are forwarded only when these symbols are modulated over an orthogonal sub-carrier. To transmit one complex data, only one carrier is used and is termed as a single carrier model. Further, $\mathrm{N}$ complex data are forwarded with the help of $\mathrm{N}$ sub-carrier and is referred to as parallel transmission and this type of transmission minimizes the delay of multipath and increases the data transmission period. But the rate of data transmission remains unchanged for both the systems. The system OFDM maintains the orthogonality among the sub-carriers by the application of inverse Fast Fourier Transform (IFFT) and a guard band is presented between each successive symbol of OFDM. The three approaches that are considered while introducing a guard band in the symbols of OFDM are namely the Cyclic prefix, cyclic suffix, and zero paddings. By introducing one guard band, the OFDM is capable of converting large frequency selective channels to a narrowband parallel flat fading channel and one channel is allotted for one subscriber. Therefore, the issue of Inter-Symbol Interference (ISI) is avoided. OFDM is primarily employed with broadband data that possess relatively large data transmission rates due to the requirement of a reduced complex equalizer and because of its significantly high resistance for fading of multipath [17].

With the help of many types of research, many approaches have been initiated for PAPR reduction. In the research area of PAPR reduction, very few remarkable frameworks are introduced and are illustrated as follows: A novel algorithm for random phase updating is explained in Ref. [18]. The phase of every sub-carrier keeps on updating with the help of an arbitrary multiplier till a PAPR exceeds below the mentioned level of threshold. The research work is based on the impact of several allocations that are employed for the phased improvement, differences in allocations of PAPR variance and PAPR mean, and analyses the iterations necessary to meet the threshold value. In this technique, the level of a threshold reduces when the phase shifts are rationalized effectively and accordingly the variance of the augmented phase is adapted. The experimental outcomes of the algorithm demonstrate relatively close concurrence. The mean power variance of the OFDM signal is lowered by $7 \mathrm{~dB}$ with the application of an arbitrary phase updating algorithm and thereby achieving better outcomes through the considerable values of threshold. A companding approach for an OFDM system is presented in [19] which is regarded as a novel algorithm for PAPR reduction. In this companding approach, the compressing polynomial present at the transmitter end is linked with the IFFT block, and the FFT block is integrated with the extended inverse function and thereby solving the mathematical equations with the help of iterative Jacobi's approach. The complexity is reduced in the proposed approach when compared to other algorithms that are designed for reducing PAPR. The SNR was found to be relatively low for the respective BER in the proposed work when compared to that of the commanding technique. Based on the complexity and execution, the number of iterations necessary for the algorithm, and the order of compressing the 
polynomial at the receiver side is identified. Thus, the above issue is solved by introducing an algorithm of selected mapping explained in Ref. [20]. Due to the presence of various parameters provided for the transmission of signal, the proposed algorithm offers better results when compared to the other SLM algorithms and OFDM chooses the parameters required for the transmitting. Real and imaginary are the main parts of OFDM. The suggested algorithm makes use of conjugate symmetry of real sequence structures by retaining perceptual computational difficulty. The proposed algorithm illustrates enhanced simulation outcomes in contrast to the traditional algorithm of SLM that is associated with MIMO-OFDM schemes. The paper [21] primarily concentrates on the application of Partial Transmit Sequence (PTS) for the reduction of PAPR. A comprehensive examination of all the stated phase factors is necessary for a traditional PTS technique. Therefore, as the sub-block increases, the processing complexity also increases gradually. Meanwhile, the traditional algorithms faced many problems therefore, a novel algorithm was presented to overcomes these issues and is based on the approach of nonlinear optimization which is also known as simulated annealing and the main objective of this framework is to reduce processing overhead by setting ideal phase factors. The experimental outcomes show that the suggested approach maintains a high reduction in PAPR by minimizing the processing cost.

To reduce significantly high PAPR for a single input single output (SISO) signals of OFDM, many techniques have been presented in this research work. The scrambling approach and signal distortion technique are the two approaches to Signal distortion. The scrambling techniques which are employed for the distortion of signal to reduce PAPR are Peak cancellation [22], clipping [23], tone reservation (TR) [24], tone injection (TI) [25], and nonlinear companding [26]. Selective mapping (SLM) approach [27], partial transmit sequence (PTS) strategy [28], coding system [29] and complement block coding approach [30]. The comprehensive research study of various techniques that are meant for the reduction of PAPR illustrates in [31]. One of the most popularly used techniques that can minimize the PAPR without modifying the signals of OFDM is SLM. Some of the newly proposed techniques presented in this research work are [33], [34], [35], and [36] which are based on SLM for the reduction of PAPR. The side information that is forwarded to the receiver side is necessary for all the techniques mentioned above but except the approach illustrated in [34]. The performance of reducing PAPR remains unchanged for both conventional SLM technique in [33] and well as the approach presented in [6]. Thus, improved performance in PAPR reduction is obtained by eliminating the side information in the SLM based technique. A novel YI research work is presented in [36], the techniques mainly utilized clipping noise for determining the best optimal set of extended symbols and outer ring hexagonal symbols. The main drawback of the TI technique is that processing cost is high since every iteration involves three IFFT operations to minimize PAPR reduction. Thus, in this paper, we develop a technique that overcomes the drawbacks of the previous approaches. Trellis-assisted constellation subset selection (TACSS) is one of the novel techniques developed for the reduction of PAPR in the OFDM system which is presented in [41]. TACSS is primarily employed for redundant bits of each data symbol for PAPR reduction and thereby lowering the spectral performance of the system. Since in the technique of TACSSS every data symbol consists of redundant bits for reducing PAPR, this reduces the system performance. The system of SISO-OFDM is developed with the application of spatial multiplexing and employs various PAPR reduction approaches, whereas MIMO-OFDM systems are space-time and frequency block coded. A novel adaptive system for TR is presented in [37] to reduce PAPR by continuously functioning on the antenna with significantly high PAPR for a MIMO-OFDM system. In [39] another technique for TR has been presented to overcome the processing cost. The inverse approach for the system of MIMO-OFDM is integrated with cross-antenna rotation to mitigate PAPR which is illustrated in [40].

In [41] an SLM system with reduced complexity is presented which utilizes an ideal set of the same magnitude to minimize the processing cost, as well as SLM, make use of additive mapping sequences [42]. The technique for reducing PAPR is not much important when compared to that of the technique of CSLM. The efficiency of PAPR reduction is minimized by presenting a system with reduced complexity that is explained in [43-45] and also the processing cost of the system is further reduced. The applicants of M4 are obtained with the functioning of M2 IFFT processes. The imaginary, as well as a real part of frequency domain input vector, are separated in the technique of linear SLM (WSLM) presented in [46] and this approach minimizes the efficiency by using M IFFT operations for PAPR reduction and thereby obtaining $4 \mathrm{M} 2$ applicants. A swapped system for SLM is presented in [47] which minimizes the 
computational complexity of traditional SLM systems. With the application of Fourier transform characteristics, the applicant signals in the $\mathrm{M}$ timedomain are partitioned as the subsequences of odd, even, imaginary odd, and imaginary even, and later these subsequence's are interchanged for obtaining M4 applicants. Furthermore, the OFDM signals that are based on time are split into real odd and even, imaginary odd and even subsequences with the help of the technique introduced in [47]. The imaginary even and imaginary odd sequences are obtained with the application of time reversal, cyclic shift, and time reversal of the cyclic shift operation techniques. Thus, the efficiency of PAPR reduction is improved by using the WSLM approach [46]. Several wireless communication systems employ orthogonal frequency division multiplexing (OFDM) technique that includes IEEE 802.11, Long-Term Evolution (LTE), and LTE Advanced systems. These systems involve reduced structuring intricacy, as well as propagation in multipath, which is reduced by providing an adaptable environment. A high peak-toaverage power ratio (PAPR) is achieved because of the non-linearity characteristic of power amplifiers. As a result, the input signal that is transmitted is distorted. The technique of reducing PAPR is one of the promising fields in the study of OFDM [47]-[50]. During the approach of clipping, the signals present at the input side are distorted deliberately so that the transmitted signal is allocated in an appropriate manner of the power amplifier. Therefore, the approach of clipping is considered as one of the suitable and efficient techniques for reducing PAPR in the system of OFDM, and the foremost disadvantage of this approach is the reduced bit error rate of a system. The approaches that are used for encoding signals are the most popular techniques employed for the reduction of PAPR. Some of the signal encoding approaches that are used are partial transmission sequence (PTS) and selective mapping (SLM) [48], [49]. In the case of the signal encoding technique, the signals are effectively reconstructed at the receiver side with the help of encoded signal generated at the receiver by the phase factor and the system efficiency reduces if the side information is not correctly obtained. Reduced processing cost is required to identify the phase factor which generates a suitable encoded sequence. Meanwhile, for reducing PAPR, a non-linear companding transformation is introduced. The Institute for Information \& communications Technology Promotion (IITP) authenticates the designed proposed framework and this institute funding is supported by the Korea government (MSIT) (No. 2014-0-00282, Advancements of 5G Mobile
Communication Technologies for Hyper-connected smart services) M. Kim and D.H. Cho are with the school of Electrical Engineering, Korea Advanced Institute of Science and Technology. W. Lee is with the Department of Information and Communication Engineering, Institute of Marine Industry, Gyeongsang National University, Republic of Korea. [50]; The bit error rate and signal distortion obtained for the proposed system corresponds to the clipping approach. In the field of natural language processing and computer vision, the approach of deep learning provides improved efficiency when compared to that of the conventional hand-crafted approaches. In the architecture of deep learning the input and output layers are integrated with several layers of perceptron's and this interconnection or link corresponds to the neurons in the human brain. Thus, to perform sensitive functions namely image identification, the technique of deep learning is one of the efficient tools and is employed in the applications of communication. In contrast to other conventional strategies, the deep learning approach provides improved performance in decoding of the channel as well encoding of the channel which is demonstrated in [51]. The identification of modulation in radio is analyzed with the application of convolution neural networks presented in [52]. Thus, with the usage of artificial network networks, the drawback of PAPR reduction is overcome and this framework is presented in [53] as well as [54]. The framework of ANN primarily focuses on reducing the computational cost of the active constellation extension (ACE) technique. The technique of active constellation extension is one of the most popularly employed approaches for PAPR reduction that is derived from the technique of clipping. But when compared to other conventional strategies, the ACE presents significantly low performance. Thus, batch normalization which is a highly developed deep learning technique is eliminated during the analysis of a shallow neural network (2 layers with 2 nodes). To reduce PAPR an autoencoder is considered which is one form of DNN and is used for denoising the corrupted data which is illustrated in [55]. Every subcarrier is given training by the DNN for the constellation demapping and mapping of symbols present in the system of OFDM. As a result, the signals transmitted in sequence provide reduced PAPR and a significantly low bit error rate. Thus, the system once trained can be employed in real-time applications with significantly low processing cost [56-58]. In this framework, there exist significantly two developments which are stated as follows: 
1. A novel reduction technique known as PRNet is suggested for PAPR and is based on the structure of a deep autoencoder. The constellation of mapping symbols and demapping symbols on every subcarrier is accomplished by the deep learning approach in the system of OFDM. Thus, the system once trained can be employed in real-time applications with significantly low processing cost and minimized PAPR reduction in the systems of OFDM.

2. The results of the suggested system are examined with the application of computer simulations. Experimental results prove that the PAPR for the system of OFDM is relatively lowered with the help of PRNet (that is the probability of PAPR is less than $0.1 \%$ when it is above $3 \mathrm{~dB}$ ) and thereby BER is maintained. In various instances, the technique of SLM which is employed for reducing PAPR offers less processing cost along with enhanced quality negotiations such as possible error rate. A substitute signal mapping technique is employed in [6] to reduce the penalty gap for the system of OFDM with help of quadrature amplitude modulation. Through phase offset mapping, the data rate, as well as the error rate, is augmented in Alamouti coded MIMOOFDM presented in [7] and at the receiver side, these phase rotation systems are re-established with a minimum Euclidian distance (MED) decoder. Further, to enhance the power performance, a parallel artificial bee colony (P-ABC) algorithm is proposed in [8]. The power reduction is carried out with the help of the SLM approach and this method can be employed for orthogonal frequency division multiplexing (OFDM) as well as for multiple-input multiple-output (MIMO)-OFDM systems. Thus, from the obtained results it is shown that the presence of the P-ABC algorithm achieves a significant reduction in PAPR and enhances the efficiency of BER with relatively low processing cost. An approach of modified Selective Codeword Shift is designed for the system of MIMO OFDM presented in [9] and with the help of interleaving approaches the multiplication of phase factors is carried out. Several adaptive methods for reducing power have been employed to accomplish relatively better computational complexity and power reduction. The signals of greater size are compressed to obtain relatively better gain for PAPR through the commanding transforms illustrated in [10] and enhanced BER performance is achieved through OOB radiation reduction. From the simulation results, it is shown that the companding transform is one of the best techniques for reducing PAPR and it is proved that it performs better than the Tanh transform. To minimize the processing cost for PAPR reduction and to narrow down the reduced performance of BER, the PTS employs few hybridization optimization algorithms which is explained in [11] and the algorithms employed are Particle Swarm Optimization (PSO) and Grey Wolf Optimizer (GWO). The ideal phase rotation parameters are chosen by PSO-GWO with the comprehensive analysis of various quality measurements. The framework described in [12] integrates both PTS and Gaussian pulse-based TR systems to reduce the PAPR with enhanced efficiency of error rate in the system of OFDM. When compared to other optimization systems the hybrid combination of various approaches provides a remarkable hardware utilization rate. In this research work, we suggest a machine learning approach that is driven by power and is based on PAPR reduction that includes three distinct systems for the system of MIMO OFDM and is stated as follows:

$>$ Reduced computational complexity is achieved through the Dynamic selection of PAPR systems over a large set of subcarriers.

$>$ The trade-off limitations associated with the traditional PAPR systems can be overcome for modulation of a high order.

With the application of direct PAPR that is based on data accessibility, the processing speed is enhanced because of the fully automated selection at every stage.

\section{Motivation}

The basic goal of the suggested framework is to accomplish reduced processing costs and to achieve a well-appropriate system by mitigating the application of PAPR characteristics in the systems of OFDM with ICI termination or without the termination of ICI. One of the significant challenges faced by the suggested work is high PAPR. Hence, in the modern approaches of reducing PAPR, we identify and assess the difficulties involved and significant developments for obtaining a realistic OFDM system. Thus, in the proposed framework the main area of interest is the examination of reducing the PAPR techniques optimally for the approaches of OFDM. Some of the popularly known systems that are used for standardizing the PAPR are tone Reservation, Coding, peak windowing, clipping, and Tone Injection. The two best suitable approaches used for minimizing PAPR are Filtering and clipping. Consequently, the filtering and clipping techniques acquire the potency to minimize PAPR at the cost of 
system performance of BER. The performance of BER decreases when there is high non-linear distortion and with help of available techniques, the PAPR can be mitigated. Therefore, filtering and clipping techniques result in distortion which is produced in the out-band and the in-band. The main objective of the suggested framework is to minimize PAPR and augment BER efficiency with the application of SLM and PTS strategies. The primary goal of the suggested framework is stated as follows: 1. Designing an effective system by examining various techniques that are employed for the reduction of PAPR and BER.

2. Designing a new hybrid technique for minimizing the PAPR and BER by integrating the SLM and PTS systems based on OFDM.

Establishing a new hybrid technique by minimizing the PAPR and BER for all the available techniques.

This paper is organized in the following manner: The advantages and implementations of several PAPR reduction techniques are explained in Section 2 and Section 3 examines the features of different PAPR approximations. The evaluation of proposed systems that are used for reducing PAPR and the performance of BER is presented in Section 4 and the last section gives the conclusion of this framework.

\section{3 HDNDYHDJH3 RZHU5 DWR 73 \$ 35 पू}

For every individual antenna, the PAPR has to be calculated in the case of a MIMO-OFDM system. As the system of OFDM is a multi-carrier, the modulated subcarriers that are stored in the time domain results in high power. The ratio of peak power to its average power is termed as PAPR and is represented as shown in equation (1).

$$
P A P R=10 \log \frac{P_{P E A K}}{P_{A V G}} d B
$$

During the process of estimation, the complex baseband is illustrated as shown in Eqn. (1) and is computed using time interval as follows;

$$
P A P R=10 \log \frac{\max |X(t)|^{2}}{E|X(t)|^{2}} d B
$$

The maximum power signal and the average power signal is indicated as $\max |X(t)|^{2}$ is and $E|X(t)|^{2}$ respectively.

Similarly, with the help of the Complementary Cumulative Distribution Function (CCDF), the PAPR functioning is estimated. The CCDF examines the consequences of surpassing PAPR in a specific PAPR threshold bound as indicated in Eqn. (3)
$C C D F(\gamma)=\operatorname{Pr}(P A P R>\gamma)=1-\left(1-e^{-\gamma}\right)^{N}$

Selective Mapping: One of the approaches that are used for minimizing the PAPR in an OFDM system is selective mapping and this is considered as a distortions-less Signal encoding technique. Further, the phase rotation is computed for every signal that is modulated and is positioned before the IFFT block while the power generated for the overall system is minimized. But the processing cost is more for the SLM technique and is enhanced exponentially with the range of subcarriers and phase sequence rate also increases in the system of OFDM. In the case of the SLM technique, several replicas of the data unit are multiplied with various phase factors as shown below:

$\mathrm{B}(\mathrm{S})=\left[\mathrm{B}^{\mathrm{S}, 1}, \mathrm{~B}^{\mathrm{S}, 2}, \mathrm{~B}^{\mathrm{S}, 3}, \ldots \mathrm{B}^{\mathrm{S}, \mathrm{M}}\right]^{\mathrm{T}}$

where the length of the phase sequence is represented as $\mathrm{M}$.

it results with $\mathrm{S}$ distinct modified data blocks and IFFT is calculated for every modified data block each related with distinct phase factors as represented in Eqn. (4)

$\mathrm{X}^{(\mathrm{s})}(\mathrm{t})=\operatorname{IFFT}\left\{\mathrm{Bs}, \mathrm{n}^{*} \mathrm{Xn}\right\}$.

Where $n$ ranges from 0 to $M$ to cover all probable dynamic ranges of modified data blocks.

Partial Transmit Sequence (PTS): The technique of PTS divides the actual block of signals into various subblocks and the subcarriers that are present in every subblock with a phase factor are phase rotated. At last, the entire PAPR is minimized due to the combination of these subblocks. Whereas the input data must be re-established at the receiver end with the application of the suitable inverse function. However, because of the detailed analysis of all probable sets of phase factors that is better is chosen but the processing cost remains significantly high. During partitioning the decomposed sub-blocks $\mathrm{X}^{(\mathrm{m})}$ is multiplier with appropriate phase factor $\mathrm{B}^{(\mathrm{m})}=\mathrm{e}^{\mathrm{j}_{\varphi}(\mathrm{v})}$, where ${ }^{\circ}(\mathrm{v})=(0,2 \pi)$. Then the integrated time-domain vectors are formulated as follows:

$$
Z=\operatorname{IFFT}\{y\}=\sum_{m=0}^{M-1} B^{(m)} \operatorname{IFFT}\left\{X^{(m)}\right\}=\sum_{m=0}^{M-1} B^{(m)} X^{(m)}
$$

Where $\mathrm{m}$ denotes complex phase factor sets which combine with the PTS as a vector shown in Eqn. (5) $\mathrm{B}^{(\mathrm{m})}=\left[\mathrm{B}^{1}, \mathrm{~B}^{2}, \mathrm{~B}^{3}, \ldots \mathrm{B}^{\mathrm{M}}\right]^{\mathrm{T}}$

The overall processing intricacy of PTS can be minimized with detailed analysis of every iteration process and consisting of few trade-offs in the efficiency of power and the phase factors $\mathrm{B}(\mathrm{m})$ is also restricted with some dynamic ranges for optimization.

\section{Hybrid SLM-PTS Techniques:}

The techniques of both SLM and PTS are necessary for the system of MIMO-OFDM to actively function 
on the antenna that is used for transmission. In this encoding technique, the signal encoding is introduced before the block of IFFT decomposition. The SLM optimized phase switched modulated data is employed for every IFFT subblocks and is employed to represent a signal with low PAPR when compared to other techniques. At this phase, the number of iterations that are required for IFFTS to minimize PAPR is reduced and the best suitable combination is selected and is depicted in figure 1 .

The processing overhead of the SLM system is now limited to $\{0, \pi\}$ with the phase selection of $\in\{1$, $-1\}$. Therefore, the selection of a phase rotation system is enhanced and as represented in Eqn (4)

$$
P A P R=\arg \min _{l=1 . . M}[P A P R(\operatorname{IFFT}(l \Theta X))]
$$

Here an enhanced result is achieved without executing all sets of combinations, i.e., $1=2 \mathrm{M}$. However, the processing intricacy increases exponentially with the increase in subcarrier number. The simulation results and the analysis of performance are achieved for two distinct sets with the subcarriers $\mathrm{N}=64$ and 128. A relatively low PAPR is chosen for the system of MIMO-OFDM and the in this case the phase factors are generated arbitrarily. The projected scheme abide of a threestage technique that assemble raw EEG signals, transform them for artefact removal, extracts features, and detects anomalies. Second, classifiers are used to classify the data, and the results are then interfaced with the FPGA. The procedure is defined in the following section.

The analysis of the proposed hybrid SLM and PTS techniques are carried out for various strategies of modulation and data transmission from 4 transceiver antennas are carried out with different conditions of noise. For every individual value of PAPR, the SVM algorithm that is based on machine learning is examined and the efficiency of a system in terms of BER and PAPR is verified. Depending on the boundary values of the threshold allotted, the reduction approaches for PAPR are chosen. Some of the preferred systems such as SLM employing SVM classifier or PTS enhances the level of efficiency for the system of MIMO-OFDM and thereby minimizes the intricacy trade-off metrics which is depicted in figure.1. $[6,7,8,9]$ are the boundary values of threshold allotted for the system and every individual value of boundary is approximated by the approach of SVM for reducing the PAPR. The below stated machine learning algorithm which is executed step by step is illustrated as follows. The steps from 1 to 3 are preferred based on the values of boundary and the network trained depending on the excepted values of PAPR and BER (these values are already defined).
The approaches such as PTS or SLM is preferred by the multi-rate SVM depending on the values of BER and PAPR for reducing their values.

\section{Machine Learning algorithm:}

Inputs: Training_Set, Group_Train, Test_Setl

Output: Trained data for reduction in PAPR

Assignment $\quad 1$ : $\quad$ PAPR_bound $=[6,7,8,9]$;

Training_Set=[PAPR_bound];

Assignment 2: Group_Train $=[1 ; 2 ; 3 ; 4]$;

Assignment 3: TestSetl=papro;

Where papro(i) $=10 * \log 10$ (peako/meano);

$$
\text { if papro }<6
$$

disp('conventional mode')for no further reduction techniques are required in PAPR

end

where peako $=\max \left(\operatorname{abs}(\right.$ time_domain_signal $\left.) .{ }^{\wedge} 2\right)$;

where

time_domain_signal=abs(ifft([S_P_DATA(i,1:32)

$\operatorname{zeros}\left(1,(\mathrm{~L}-1)^{*} \mathrm{Nt}\right)$ S P DATA(i,33:64)]));

Step:1 Find the uniqueness values in Group_Train data

$$
\text { Calculate the length on the Step: } 1
$$

Step:2 if (Group_Train) has unique values the Assign Test_Setl=Group_Train;

Step:3 Create empty matrix to store the trained data of Traning_set

Group_Train

For loop from $\mathrm{k}=1$ to length of

$$
\text { If(G1vAll }
$$

$=$ Group_Train $=$ length $(\mathrm{k}))$ then

Train for models $(\mathrm{k})=$ svmtrain(TrainingSet(:,1:4),G1vAll,'kern el_function','rbf');

It is to check current class is 1 or 0

Step:4 classify and make sure whether PTS is carefully chosen or SLM is designated for further reduction in PAPR which is based on the value of existing PAPR

Else

$\operatorname{Return}(\mathrm{j})=\mathrm{k}$;

end 


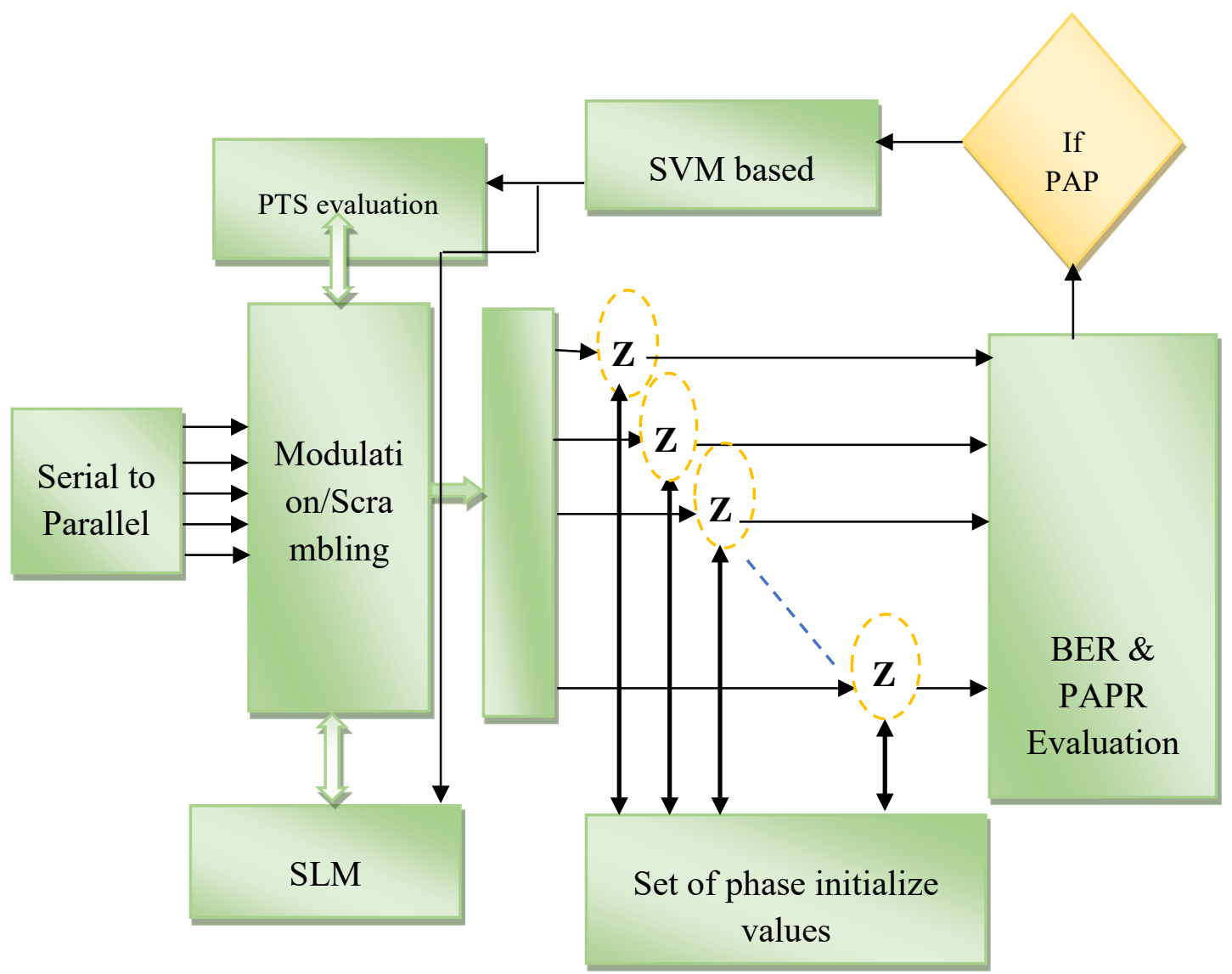

Fig 1: Hybrid SLM-PTS PAPR reduction scheme

\section{Simulation Results}

The suggested machine learning technique which is based on a hybrid PAPR selection system is efficiently modeled in this section of the framework. This part of the paper also explains the ability to approximate an efficient set of parameters with minimum processing cost for PAPR reduction. With the available MIMO OFDM techniques, the comparison of BER and PAPR reduction outcomes are carried out. The simulation techniques such as partial transmit sequence, clipping, and Selective mapping are illustrated in this research paper. The intricacy trade-off is considered as one of the benefits of all the three approaches that are designed for the reduction of PAPR and if the upper threshold value is less than the real power estimated then the Hybrid SLM-PTS approach is chosen otherwise if the lower threshold value is greater than the real estimated power then all three techniques are excluded. From the experimental outcomes, it is shown that the efficiency of PAPR is augmented for a hybrid set of distinct IFFT values due to the employment of the CCDF graph. Higher values of IFFT result in improved SLM to mitigate the PAPR which is stated previously and is therefore well proven in these findings. The entire processing time is minimized with the application of a technique that is based on machine learning even though the approaches chosen for PAPR reduction is probabilistic. The analysis of the MIMO OFDM signal for two different levels of sub-carrier is presented in Fig. 2 and Fig 3. These figures also depict the CCDF of BER and PAPR.

The conventional purpose of using the MUD method is to reduce the co-channel interference. However, in recent years several methodologies are invented in order to develop a MUD that can perform signal detection with the least complexity overhead. In recent days MUD technique is accomplished using fast Fourier transform (FFT) by converting received symbols into frequency domain as overlapping the blocks and suppresses the interference by deriving 
weights for each symbol from the minimum mean square error (MMSE) criterion which retain at low complexity irrespective of user rate. In general, linear MMSE MUD cannot perform well over a multi-user environment which is highly sensitive to co-channel interferences since inter-user interference has no degrees of freedom.

In this section, both MATLAB simulations and digital architecture implementations are carried out to demonstrate the error performance and design complexity respectively, of the proposed multi beamformer and signal detector. The following facts can be observed: (i) All beams formulated with equal gain using FFT transforms are orthogonal to each other and complexity is decreased exponentially as N (FFT point) increases. (ii) Our optimized ML matches with the analytical ML BER very well in moderate and low SNR regimes and ends with the least discrimination when BER is below 10-3 and explored that our optimal ML has major advantages in terms of complexity and data rate efficiency. In addition, FPGA hardware synthesis results can exploit the finite beneficiary measurements of our radix factorized FFT and optimized ML detectors.

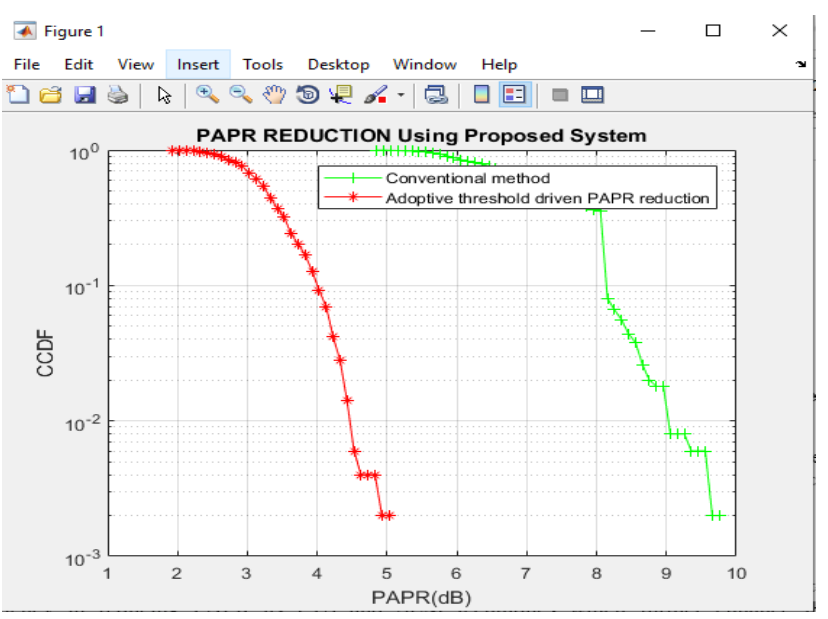

Fig 2: PAPR performance comparison of proposed threshold driven mode selection with the conventional model

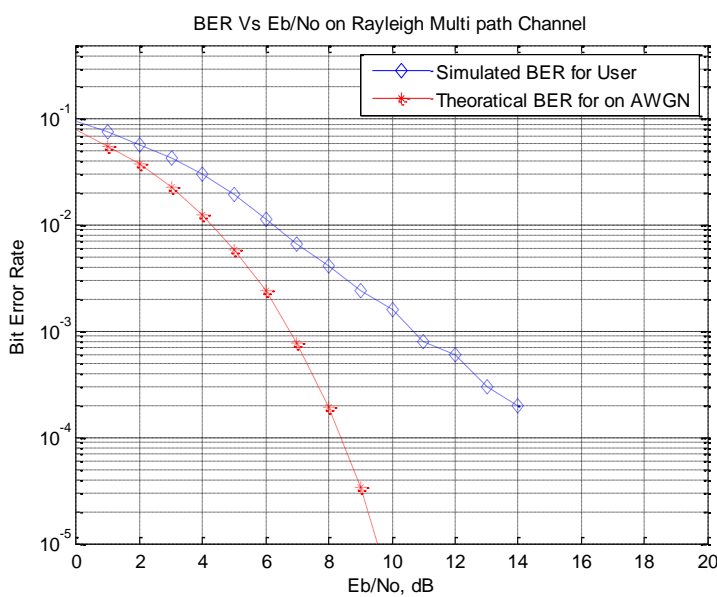

Fig 3: BER error rate performance analyses of proposed threshold driven mode selection over theoretical performance.

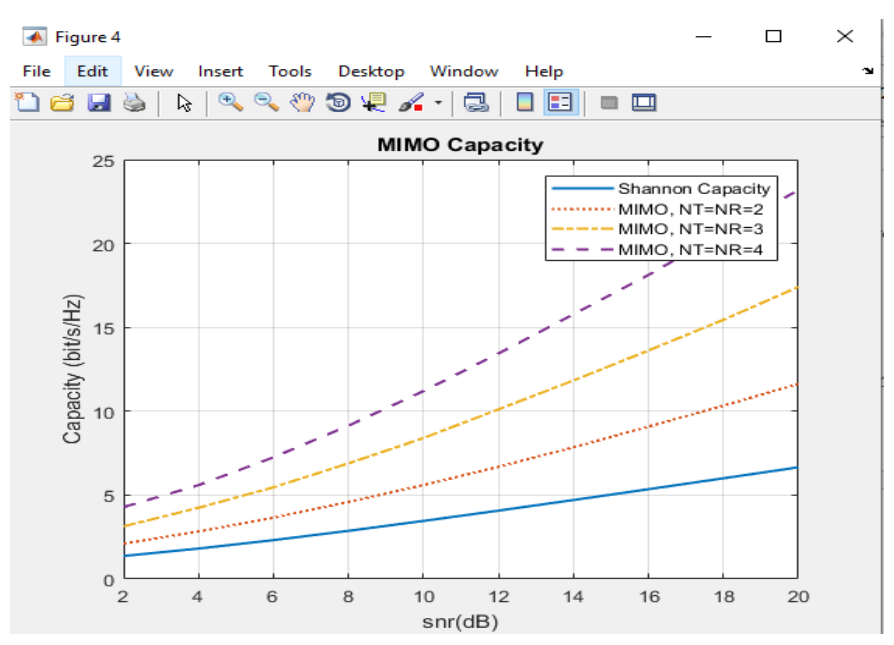

Fig.4. Performance improvement in SNR and Channel Capacity of proposed wireless communication system with Machine learning

The analysis of the hybrid techniques which are based on MIMO-OFDM and SVM based machine learning algorithms for two different levels of subcarrier, the Signal-to-Noise Ratio (SNR) and capacity improvement for different number of transmitter antenna's and receiver antenna's are presented in Fig. 4. This results are compared with Shannon capacity and it is proven that the proposed system has increased both SNR and channel capacity during the transmission of packets. Every channel capacity is estimated with respect to MSE for the different techniques in both theoretical and practically as shown in Fig.5. 


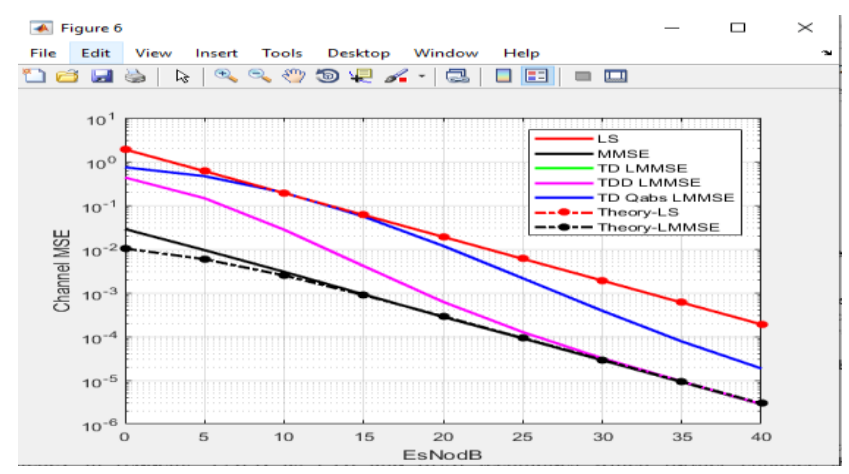

Fig.5. Reduction in channel MSE for different techniques in both theoretical and practical results

\section{Conclusion}

In this framework, we make use of a hybrid technique of SLM-PTS to provide better efficiency in reducing PAPR as PTS and SLM techniques which further enhance the performance of PAPR from communication overhead. Depending on the PAPR and BER computation, any one of the above techniques is chosen for the traditional subcarrier mapping in MIMO-OFDM systems for reducing PAPR. With the help of standardized easy process which is identical to the technique based on machine learning, the possibility of the system which is based on an automated training network offers enhanced transmission rate of data for reducing PAPR and thereby make use of the probabilistic benefits of every PAPR system and choose a pre-defined tradeoff metric. Based on the obtained results, the proposed system has been reduced the MSE and BER and also improved the performance level of channel capacity and SNR.

\section{References}

[1]. Latinovic, Z., and Bar-Ness, Y., 2006. SFBC MIMO-OFDM top to-average force proportion decrease by polyphase interleaving and reversal. IEEE correspondences letters, 10(4), pp.266268.

[2]. Shin, J.O.O.N.W.O.O. furthermore, Seo, B.A.N.G.W.O.N., 2014. PAPR Reduction Scheme for MISO and MIMO OFDM Systems with Limited Feedback. WSEAS Transactions on Communications, 13, pp.355-362.

[3]. Han, S.H. furthermore, Lee, J.H., 2004. Changed chosen planning method for PAPR decrease of the coded OFDM signal. IEEE exchanges on communicating, 50(3), pp.335-341.

[4]. Han, S.H., and Lee, J.H., 2004. PAPR decrease of OFDM signals utilizing a diminished intricacy
PTS procedure. IEEE Signal Processing Letters, 11(11), pp.887-890.

[5]. Wang, S.H., and Li, C.P., 2009. A lowmultifaceted nature PAPR decrease conspires for SFBC MIMO-OFDM frameworks. IEEE Signal Processing Letters, 16(11), pp.941-944.

[6]. Jeon, H.B., No, J.S. what's more, Shin, D.J., 2011. A low-intricacy SLM conspires utilizing added substance planning successions for PAPR decrease of OFDM signals. IEEE Transactions on Broadcasting, 57(4), pp.866-875.

[7]. Jiang, T., Ni, C., and Guan, L., 2013. An epic stage counterbalance the SLM plot for PAPR decrease in Alamouti MIMO-OFDM frameworks without side data. IEEE signal handling letters, 20(4), pp.383-386.

[8]. Taşpınar, N. furthermore, Yıldırım, M., 2015. A tale equal fake honey bee settlement calculation and its PAPR decrease execution utilizing the SLM conspire in OFDM and MIMO-OFDM frameworks. IEEE Communications Letters, 19(10), pp.1830-1833.

[9]. Abdullah, E., Idris, A., and Saparon, A., 2017. PAPR decrease utilizing scs-slm strategy in stfbc Mimo-OFDM. ARPN J Eng Appl Sci, 12(10), pp.3218-21.

[10]. Mounir, M. what's more, El_Mashade, M.B., 2019. On the determination of the best companding strategy for PAPR decrease in OFDM frameworks. Diary of Information and Telecommunication, 3(3), pp.400-411.

[11]. Kumar, P.R., Naganjaneyulu, P.V., and Prasad, K.S., 2019. Half breed PS-GW advanced PTS plot for PAPR decrease in OFDM framework. IET Communications, 13(18), pp.2996-3002.

[12]. Vijayalakshmi, M. what's more, Reddy, K.R., 2020. A successful crossover approach for PAPR decrease in MIMO-OFDM. Simple Integrated Circuits and Signal Processing, 102(1), pp.145-153

[13]. Amritpal Singh.et.al, "Top to average force proportion decrease in OFDM framework utilizing half breed method", Optik 127 (2016) 3368-3371,

http://dx.doi.org/10.1016/j.ijleo.2015.12.105 0030-4026, 2015 Elsevier Gmb

[14]. H.A.S. Namitha, S.M. Sameer, A bandwidthefficient selective mapping technique for the PAPR reduction in spatial multiplexing MIMOOFDM wireless communication system, Physical Communication (2017), https://doi.org/10.1016/j.phycom.2017.09.009.

[15]. Vaiyamalai Sudha.et.al, "Low multifaceted nature PAPR decrease in SLM-OFDM 
framework utilizing time-space arrangement detachment", Alexandria Engineering Journal (2018) 57, 3111-3115, https://doi.org/10.1016/j.aej.2017.11.006, 11100168 _ 2018 Faculty of Engineering, Alexandria University. Creation and facilitating by Elsevier B.V.

[16]. Minhoe Kim.et.al, "A Novel PAPR Reduction Scheme for OFDM System dependent on Deep Learning", DOI 10.1109/LCOMM.2017.2787646, IEEE Communications Letters, 1089-7798, 2017 IEEE.

[17]. Amritpal Singh.et.al, "Top to average force proportion decrease in OFDM framework utilizing hybridtechnique", Optik 127 (2016) 3368-3371, http://dx.doi.org/10.1016/j.ijleo.2015.12.105 0030-4026, 2015 Elsevier

[18]. Homayoun Nikookar, Knut Sverre Lidsheim, Random stage refreshing algorithmfor OFDM transmission with low PAPR, IEEE Trans. Broadcast. 48 (2) (2002)123-128

[19]. Iraj Hosseini, Mohammad Javad Omidi, Keyvan Kasiri, Alireza Sadri, P. GlennGulak, PAPR decrease in OFDM frameworks utilizing polynomial-based compressingand iterative growing, IEEE 4 (2006) 333-336, 142440469X

[20]. Jing Gao, Jinkuan Wang, Zhibin Xie, Peak to average force proportion decrease forMIMOOFDM frameworks with deteriorated chosen planning, Int. J. Inf. Syst. Sci.3 (3-4) (2009) 572-580.

[21]. Tao Jiang, Weidong Xiang, Paul C. Richardson, Jinhua Guo, Guangxi Zhu, PAPRreduction of OFDM signals utilizing incomplete send groupings with low computational unpredictability, IEEE Trans. Broadcast. 53 (3) (2007) 719-724

[22]. S. Cha, M. Park, S. Lee, K.- J. Blast, D. Hong, another PAPR decrease procedure for OFDM frameworks utilizing progressed top windowing technique, IEEE Trans. Purchaser Electronics, 54 (2) (May 2008) 405-41

[23]. D. Wulich, L. Goldfeld, Reduction of pinnacle factor in symmetrical multicarrier adjustment by abundancy restricting and coding, IEEE Trans. on Commun. 47 (1) (1999) 18-2

[24]. D. Guel, J. Palicot, Y. Loue, Tone reservation procedure dependent on mathematical strategy for symmetrical recurrence division multiplexing top to-average force proportion decrease, IET Commun., 4 (17) (Nov. 2010) 2065-2073.
[25]. J. Hou, C. Tellambura, J. Ge, Tone infusion for PAPR decrease utilizing equal unthinkable inquiry calculation in OFDM frameworks, in Proc. IEEE Global Telecommun. Conf. (GLOBECOM), Dec. 2012, pp. 4899-4904.

[26]. T. Jiang, G. Zhu, Nonlinear companding change for decreasing top to-average force proportion of ofdm signals, IEEE Transactions on Broadcasting 50 (3) (Sep. 2004) 342-346.

[27]. R.W. B"auml, R. F. Fischer, J. B. Huber, Reducing the top to-average force proportion of multicarrier regulation by chose planning, IET Electronics Lett., 32 (22) (Oct. 1996) 20562057.

[28]. P. Varahram, W. F. Al-Azzo, B. M. Ali, A low unpredictability fractional communicate grouping plan by utilization of fakers signals for PAPR decrease in OFDM frameworks, IEEE Trans. Consum. Electron. 56 (4) (Nov. 2010) 2416-2420.

[29]. T. Jiang, X. Li, Using wellspring codes to control the top to-average force proportion of OFDM signals, IEEE Trans. on Vehicular Tech., 59 (8) (Oct. 2010) 3779-3785.

[30]. T. Jiang, G. Zhu, Complement block coding for the decrease in top to-average force proportion of OFDM signals, IEEE Communications Magazine 43 (9) (Sep. 2005) S17-S22.

[31]. T. Jiang, Y. Wu, A diagram: Peak-to-average force proportion decrease procedures for ofdm signals, IEEE Transactions on communicating 54 (2) (Jun. 2008) 257-268.

[32]. H.- B. Jeon, J.- S. No, D.- J. Shin, A lowintricacy SLM plot utilizing added substance planning groupings for PAPR decrease of OFDM signals, IEEE Trans. Broadcast. 57 (4) (Dec. 2011) 866-875.

[33]. T. Jiang, C. Ni, L. Guan, A tale stage balance SLM plot for PAPR decrease in AlamoutiMIMO-OFDM frameworks without side data, IEEE signal handling letters 20 (4) (Apr.2013) 383-386

[34]. J.- Y.Woo, H. S. Joo, K.- H. Kim, J.- S. No, D.- J. Shin, PAPR examination of class-III SLMscheme dependent on the fluctuation of the relationship of option OFDM signal arrangements, IEEE Commun. Lett. 19 (6) (Jun. 2015) 989-992

[35]. N. Tas,pınar, M. Yıldırım, A Novel Parallel Artificial Bee Colony Algorithm and Its PAPR Reduction Performance Using SLMScheme in OFDMandMIMO-OFDMSystems, IEEE Commun. Lett. 19 (10) (Oct. 2015) 1830-183 
[36]. J. Hou, X. Zhao, F. Gong, F. Hui, J. Ge, PAPR and PICR Reduction of OFDM Signals with Clipping Noise-based Tone Injection Scheme PP (99) (Mar. 2016) 1-11.

[37]. R. Yoshizawa, H. Ochai, Trellis-Assisted Constellation Subset Selection for PAPR Reduction of OFDM Signals, IEEE Transactions on Vehicular Technology, DOI: 10.1109/TVT.2016.2572139

[38]. C. Ni, Y. Mama, T. Jiang, A tale versatile tone reservation conspire for papr decrease in largescale multi-client mimo-ofdm frameworks, IEEE Wireless Communications Letters (5) (Oct. 2016) 480-483

[39]. X. He, T. Jiang, G. Zhu, A tale improved tone reservation joined with cross recieving wire pivot and reversal to lessen papr for mimo-ofdm framework, Wireless Communications and Mobile Computing 11 (10) (Oct. 2011) 14061414.

[40]. Y.- L. Lee, Y.- H. You, W.- G. Jeon, J.- H. Paik, H.- K. Tune, Peak-to-average force proportion in MIMOOFDM frameworks utilizing particular planning, IEEE Commun. Lett. 7 (12) (Dec. 2003) 575-577

[41]. E. S. Hassan, S. E. El-Khamy, M. I. Dessouky, S. A. El-Dolil, F. E. A. El-Samie, Peak-to-average force proportion decrease in space-time block coded multi-input multi-yield symmetrical recurrence division multiplexing frameworks utilizing a little overhead particular planning plan, IET J. Commun. 3 (10) (Feb. 2009) 1667-1674.

[42]. Chih-Peng Li, Sen-Hung Wang, Chin-Liang Wang, Novel low unpredictability SLM plans for PAPR decrease in OFDM frameworks, IEEE Trans. Signal Process. 58 (5) (2010) 2916-2921

[43]. H.B. Jeon, J.S. No, D. Shin, J, A lowintricacy SLM conspire utilizing added substance planning successions for PAPR decrease of OFDM signals, IEEE Trans. Broadcasting 57 (4) (2011) 866-875.

[44]. Abolfazl Ghassemi, T. Aaron Gulliver, Partial specific planning OFDM with low multifaceted nature IFFTs, Commun. Lett., IEEE 12 (1) (2008) 4-6.

[45]. Chin-Liang Wang, Ming-Yen Hsu, Yuan Ouyang, A low unpredictability top to-average force proportion decrease procedure for OFDM frameworks, Global Telecommunications Conference, 2003.GLOBECOM'03 4 (2003)

[46]. S.H. Wang, J.C. Sie, C.P. Li, Y. Chen, F, A low-intricacy PAPR decrease conspire for OFDMA uplink frameworks, IEEE Trans.
Remote Communication. 10 (4) (2011) 12421251

[47]. Robert FH Fischer, Widely-direct chosen planning for top to average force proportion decrease in OFDM, Electron. Lett. 43 (14) (2007)

[48]. L. Yang, Y.M. Siu, K.K. Soo, S.W. Leung, S.Q. Li, Low intricacy PAPR decrease procedure for OFDM frameworks utilizing altered broadly straight SLM plot, AEU-Int. J. Electron. Commun. 66 (12) (2012) 1006-1010.

[49]. Lin Yang, Hu. Wu-Jun, Kwok-Kai Soo, Yun-Ming Siu, Swapped SLM plot for lessening PAPR of OFDM frameworks, Electron. Lett. 50 (22) (2014) 1608-1609.

[50]. T. Hwang, C. Yang, G. Wu, S. Li, G.Y. Li, OFDM and its remote applications: a review, IEEE Trans. Veh. Technol. 58 (4) (2009) 16731694.

[51]. Seung Hee Han, Jae Hong Lee, A diagram of top to-average force proportion decrease strategies for multicarrier transmission, IEEE Wirel. Commun. 12 (2) (2005) 56-

[52]. Hideki Ochiai, Hideki Imai, Performance investigation of purposely cut OFDM signals, IEEE Trans. Commun. 50 (1) (2002) 89-101.

[53]. Xiaodong Li, Leonard J. Cimini Jr, Effects of cutting and sifting on the exhibition of OFDM, IEEE 47th Vehicular Technology Conference (1997)

[54]. Athinarayanan Vallavaraj, Brian G. Stewart, David K. Harrison, An assessment of changed 1Law companding to diminish the PAPR of OFDM frameworks, AEU-Int. J. Electron. Commun. 64 (9) (2010) 844-857.

[55]. V.P. Jime'nez, M.J. Garc1'a, M.P. Sa' nchezFerna' ndez, A.G. Task force, Efficient usage of reciprocal Golay successions for PAR decrease and forward blunder revision in OFDM-based WLAN frameworks, AEU-Int. J. Electron. Commun. 62 (9) (2008 Oct 1) 683-69

[56]. J.A. Davis, J. Jedwab, Peak-to-mean force control in OFDM, Golay correlative arrangements, and Reed-Muller codes, IEEE Trans. Inf. Hypothesis 45 (7) (1999) 2397-

[57]. Robert W. Ba" uml, F.H.Fischer. Robert, B.Huber. Johannes, Reducing the top to-average force proportion of multicarrier balance by chosen planning, Electron. Lett. 32 (22) (1996) 2056-2057

[58]. J.Leonard. Cimini Jr, Nelson R. Sollenberger, Peak-to-average force proportion decrease of an OFDM signal utilizing incomplete communicates arrangements, IEEE Commun. Lett. 4 (3) (2000) 86-88. 


\section{Creative Commons Attribution License 4.0} (Attribution 4.0 International, CC BY 4.0)

This article is published under the terms of the Creative Commons Attribution License 4.0

https://creativecommons.org/licenses/by/4.0/deed.en_US 